\title{
Large-Scale Models of Radiation Chemistry and the Principle of Detailed Balancing
}

David M. Stanbury* \& Jordan Harshman*

Dept. of Chemistry and Biochemistry

Auburn University, Auburn, AL 36849

Supporting Information

Code for DETBAL 1.0 
Instructions for use:

Download and install the R application, avaliable at https://www.r-project.org

Download and install the RStudio application, available at https://www.rstudio.com

Download and install packages within R: pracma, 1 inprog, shiny.

Copy and paste the code given below into a new script.

Within RStudio click the Run Document button.

\section{Insta11}

Code available on GitHub: jordanharshman/DETBAL

title: "Reaction Loop Check"

author: "Jordan Harshman"

date: "February 4, 2019"

runtime: shiny

output: htm1_document

$---$

$\cdots\{r$ setup, include $=$ FALSE $\}$

knitr: :opts_chunk\$set $($ echo $=$ FALSE)

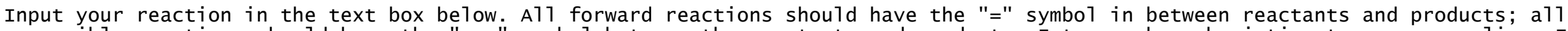

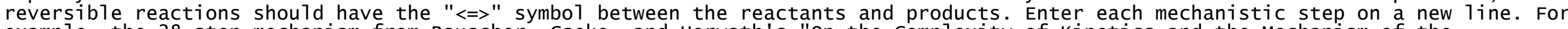
example, the 28-step mechanism from Rauscher, Cseko, and Horvath's "On the complexity of Kinetics and the Mechanism of the Thiosulfate - Periodate Reaction" in Inorganic Chemistry (2011, vol. 50, 5793-5802) would be entered as so:

$\mathrm{S} 2 \mathrm{O} 32-+\mathrm{IO} 4-+\mathrm{H}+<=>\mathrm{S} 2 \mathrm{O} 3 \mathrm{OH}-+\mathrm{IO} 3-<\mathrm{br} />$

$\mathrm{IO} 4-+\mathrm{S} 2 \mathrm{O} 3 \mathrm{OH}-+\mathrm{H} 2 \mathrm{O}=\mathrm{HOI}+2 \mathrm{SO} 42-+2 \mathrm{H}+\langle\mathrm{br} /\rangle$

$\mathrm{IO} 4-+\mathrm{S} 2 \mathrm{O} 3 \mathrm{OH}-+\mathrm{H} 2 \mathrm{O}=2 \mathrm{HSO}-+\mathrm{IO} 3-+\mathrm{H}+\langle\mathrm{br} /\rangle$

$\mathrm{S} 2 \mathrm{O} 32-+\mathrm{S} 2 \mathrm{O} 3 \mathrm{OH}-+\mathrm{H}+=\mathrm{S} 4062-+\mathrm{H} 2 \mathrm{O}<\mathrm{br} />$

$\mathrm{S} 2 \mathrm{O} 32-+\mathrm{IO} 3-+2 \mathrm{H}+<=>\mathrm{S} 2 \mathrm{O} 3 \mathrm{OH}-+\mathrm{HIO} 2<\mathrm{br} />$

$\mathrm{IO} 4-+\mathrm{HIO} 2=2 \mathrm{IO} 3-+\mathrm{H}+<\mathrm{br} />$

$\mathrm{IO} 4-+\mathrm{HOI}=\mathrm{IO}-+\mathrm{HIO} 2<\mathrm{br} />$
$\mathrm{IO} 4-+\mathrm{I}-+\mathrm{H}+=\mathrm{IO} 3-+\mathrm{HOI}<\mathrm{br}$

$\mathrm{IO} 3-+\mathrm{I}-+2 \mathrm{H}+<=>\mathrm{I} 2 \mathrm{O} 2+\mathrm{H} 2 \mathrm{O}<\mathrm{br} />$

$\mathrm{I} 2+\mathrm{I} 2 \mathrm{O} 2+2 \mathrm{H} 2 \mathrm{O}=2 \mathrm{HIO} 2+2 \mathrm{I}-+2 \mathrm{H}+<\mathrm{br} />$

$\mathrm{HIO} 2+\mathrm{I}-+\mathrm{H}+=2 \mathrm{HOI}<\mathrm{br} />$

$\mathrm{I} 2+\mathrm{H} 2 \mathrm{O}<=>\mathrm{HOI}+\mathrm{I}-+\mathrm{H}+<\mathrm{br} />$

$\mathrm{I} 2+\mathrm{I}-<=\mathrm{I} 3-<\mathrm{br} />$

$\mathrm{HIO} 2+\mathrm{I} 2+\mathrm{H} 2 \mathrm{O}=\mathrm{IO} 3-+2 \mathrm{I}-+3 \mathrm{H}+<\mathrm{br} />$

$\mathrm{S} 2032-+\mathrm{I} 2=\mathrm{S} 203 \mathrm{I}-+\mathrm{I}-<\mathrm{br} />$

$\mathrm{S} 2 \mathrm{O} 3 \mathrm{I}-+\mathrm{H} 2 \mathrm{O}=\mathrm{S} 2 \mathrm{O} 32-+\mathrm{HOI}+\mathrm{H}+<\mathrm{br} />$

$\mathrm{IO} 4-+\mathrm{S} 2 \mathrm{O} 3 \mathrm{I}-+2 \mathrm{H} 2 \mathrm{O}=2 \mathrm{SO} 42-+\mathrm{I}-+\mathrm{HOI}+3 \mathrm{H}+\langle\mathrm{br} /\rangle$

$\mathrm{S} 2032-+\mathrm{HOI}=\mathrm{S} 2 \mathrm{O} 3 \mathrm{OH}-+\mathrm{I}-<\mathrm{br} />$

$\mathrm{S} 2 \mathrm{O} 3 \mathrm{OH}-+\mathrm{S} 2 \mathrm{O} 3 \mathrm{I}-=\mathrm{S} 4062-+\mathrm{HOI}<\mathrm{br} />$

$\mathrm{HSO3}-+\mathrm{I} 2+\mathrm{H} 2 \mathrm{O}=\mathrm{SO} 42-+2 \mathrm{I}-+3 \mathrm{H}+<\mathrm{br} />$

$\mathrm{HSO3}-+\mathrm{HOI}=\mathrm{SO} 42-+\mathrm{I}-+2 \mathrm{H}+<\mathrm{br} />$

$\mathrm{IO} 4-+\mathrm{HSO}_{-}-=\mathrm{SO} 42-+\mathrm{IO} 3-+\mathrm{H}+\langle\mathrm{br} /\rangle$ 
$\mathrm{S} 2 \mathrm{O} 32-+\mathrm{HIO} 2+\mathrm{H}+=\mathrm{S} 2 \mathrm{O} 3 \mathrm{OH}-+\mathrm{HOI}<\mathrm{br} />$

$2 \mathrm{~S} 2 \mathrm{O} 3 \mathrm{OH}-+\mathrm{H} 2 \mathrm{O}=\mathrm{S} 2 \mathrm{O} 32-+2 \mathrm{HSO} 3-+2 \mathrm{H}+<\mathrm{br} />$

$\mathrm{S} 2 \mathrm{O} 3 \mathrm{OH}-+\mathrm{IO} 3-+\mathrm{H} 2 \mathrm{O}=2 \mathrm{HSO} 3-+\mathrm{HIO} 2<\mathrm{br} />$

$\mathrm{S} 4062-+\mathrm{I} 2 \Leftrightarrow \mathrm{S} 406 \mathrm{I}-+\mathrm{I}-<\mathrm{br} />$

$\mathrm{S} 406 \mathrm{I}-+\mathrm{H} 2 \mathrm{O}=\mathrm{S} 2 \mathrm{O} 3 \mathrm{OH}-+\mathrm{S} 2 \mathrm{O} 3 \mathrm{I}-+\mathrm{H}+<\mathrm{br} />$

$\mathrm{I}-+\mathrm{S} 406 \mathrm{I}-=2 \mathrm{~S} 203 \mathrm{I}-<\mathrm{br} />$

․ $\{r$ input, warning=FALSE $\}$

\# load required packages

suppressmessages (1 ibrary (pracma))

suppressmessages (1ibrary (1 inprog))

suppressmessages (1ibrary(shiny))

\# place initial text box for reaction input

textAreaInput("rxn", "Mechanism:", width $=500$, height $=250$ )

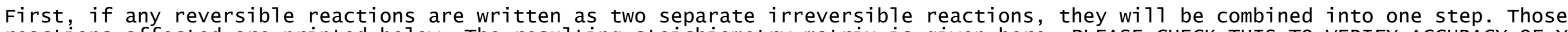

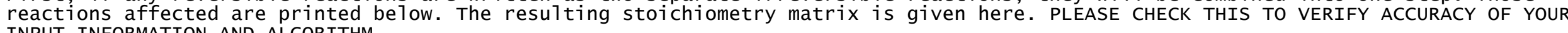
INPUT INFORMATION AND ALGORITHM.

$\cdots\{r$ rxnmat, warning=FALSE

\# FUNCTIONS:

revrxnmessage: This is a function scans for reversable reactions that were written as two irreversable reactions but never reported as one reversable reaction. It will find them, remove one of them, than then change the other to an equilibrium expression so that the rest of the code wil1 run correctly. This section only leads to the printed message that appears and does not affect the rest of the code.

revrxnrem: This is a function scans for reversable reactions that were written as two irreversable reactions but never reported as one reversable reaction. It will find them, remove one of them, than then change the other to an equilibrium expression so that the rest of the code will run correctly. This section actually replaces the equilibrium expressions in the main reaction mechanism so that it can be used in later code.

rxnmat: This is a function takes the input reaction mechanism and converts it into a reaction matrix

revrxnmessage $<-$ reactive $(\{$

rxnmech <- input\$rxn \# read the input mechanism reactively

\# convert to data frame

rxnmech <- data.frame(Steps = unlist(strsplit(rxnmech, "\n")[[1]]),

stringsASFactors $=$ FALSE)

indreacta11 <- matrix(rep("BLANK", 10*nrow(rxnmech)), nrow = nrow(rxnmech)) \# store a11 reactants

indproda11 <- matrix(rep("BLANK", 10*nrow(rxnmech)), nrow = nrow(rxnmech)) \# store a11 products

\# The for loop that follows populates the previously created blank matrices for storing reactants and products for (j in 1:nrow(rxnmech)) \{

reactprod <- data.frame(matrix(strsplit(rxnmech $[j],, "<=>=")[[1]]$, ncol = 2),

stringsAsFactors = FALSE) \# split into reactants and products

names(reactprod) <- c("Reactants", "Products") \# rename variables

indreact <- matrix(gsub(" ", "", strsplit(reactprod\$Reactants[1], " \\+ ") [[1]]), nrow = 1) \# split reactants indreact <- cbind(indreact, matrix(rep("BLANK", 10 - ncol(indreact)), nrow = 1)) \# fil1 in empty cel1s with BLANK indreacta $11[j]<$,- indreact $[1$,$] \# write the row to the storage vector$

\# same as above with products instead of reactants

indprod <- matrix(gsub(" ", "", strsplit(reactprod\$Products $[1], " \backslash \backslash+")[[1]])$, nrow = 1) 
indprod <- cbind(indprod, matrix(rep("BLANK", $10-$ ncol(indprod)), nrow = 1)) \} indproda $11[j]<$,- indprod $[1$,

indspecies <- matrix(rep("BLANK", 10*nrow(indproda11)), ncol = 10) \# store individual species

\# The for loop that follows populates the previously created blank matrix for storing all species

for (i in 1:nrow(indproda11))

react <- indreacta 1$][i],[i$ dindreacta $11[i] !=$, "BLANK"] \# capture reactants

prod <- indproda11[i,][indproda11[i,]!= "BLANK"] \# capture products

species <- c(react, prod) \# combine \}

indspecies $[i, 1: 1$ ength(species) $]<-$ species

\# write to blank storage matrix

revrows <- numeric(0) \# store the reversable rows

\# The for loop that follows populates searches for reversable reactions written as two irreversable reactions

for $(i$ in 1:nrow(indspecies) $)\{$
tomatch $<-$ indspecies $[i$,

\# see if this " $i$ " row of reactants and products matches any other row

\# The for loop that follows checks each " $i$ " row against the other " $j$ " rows

for ( $j$ in 1:nrow(indspecies)) \{

$i f(j==i)\{$

NULL \# skip if $i$ is equal to $j$ (don't campare it to itse1f)

\}else \{

doesmatch <- match(indspecies[j,], tomatch) \# check if anything in $i$ equals $j$

doesmatch2 <- match(tomatch, indspecies $[j$,$] ) \# check if anything in j$ equals $i$

$x<-$ sum(doesmatch, doesmatch2)

\# add them up

if (! is.na(x))

revrows $[i]<-j$ \# if reactants and products are exactly the same, " $x$ " wi11 be numeric;

\# if even one reactant/product are different, an NA wil1 be generated and sum(x) wi11 be NA;

\}

\# write this reaction to the storage vector if it's numeric (if it's a reversable reaction)

\}

if (length (revrows) $==0)\{$

revrxnsist <- NULL \# if no reversable reactions were detected, there is no list to populate \}else \{

\# the for loop that follows just checks for any NAs that slipped in during the process and removes this row

for ( $i$ in 1: length(revrows)) \{

if(! is.na(revrows[i]) \& is.na(revrows $[$ revrows $[i]]))$

\} revrows $[i]<-N A$

revrxns1st $<-$ data.frame $(r \times n 1=$ numeric $(0)$

rxn2 = numeric(0)) \# store the list of reactions that got combined as one reversable reaction

\# the for loop that follows populates the list of pair of reactions that got concatenated into one reversable reaction

for ( $i$ in 1: length (revrows) \{

if (! is.na(revrows [i]) \{

$x<-$ data.frame(rxn1 $=i, \operatorname{rxn2}=$ revrows[i]) \# if it's not NA, write reaction 1 and 2 into $x$ object

\# add this row to the previously created blank data frame 
revrxnslst\$Combo <- "BLANK" \# create a column of their combination

\# the for loop that follows populates the previously created blank column

for ( $i$ in 1:nrow(revrxnslst)) \{

rxn1 <- revrxnslst\$rxn1[i] \# temporarily store reaction 1

rxn2 <- revrxns1st\$rxn2[i] \# temproarily store reaction 2

rxns <- $c(r \times n 1, r \times n 2)[\operatorname{order}(c(r \times n 1, r \times n 2))]$ \# combine them by order of reaction 1 then 2 \}

revrxnslst\$Combo[i] <- paste(rxns, collapse = " ") \# concatenate the two reactions together into the blank variable

revrxns1st <- revrxns1st[duplicated(revrxns1st\$Combo), ] \# remove dup1icated rows

revrxnslstmsg <- revrxnslst \# duplicate this object to be used later

$x$ <- data.frame (matrix(c(rxnmech[revrxns1stmsg\$rxn1,], rxnmech[revrxns1stmsg\$rxn2,]),

ncol $=2$ ), stringsAsFactors = FALSE) \# convert reactions list to matrix

names $(x)<-c(" s t e p 1 "$, "step2") \# rename variables

revrxns1stmsg <- cbind(revrxnsistmsg, $x)[,-3]$ \# bind this to the previously stored data frame

\# the for loop that follows checks to see if an equilibrium sign is already present for each row; if so, it's ignored;

\# if not, it replaces the = sign with the $\Leftrightarrow=>$ sign

for ( $i$ in revrxns1st $\$ r \times n 2)$ \{

if (length $($ grep $("<=>", \operatorname{rxnmech}[i]))=,=1)\{$

NULL \# ignore

\}else\{ \}

rxnmech <- as.data.frame(rxnmech[-revrxns]st\$rxn1,], stringsAsfactors = FALSE) \# fina11y, rewrite rxnmech to be used 1ater \}

names(rxnmech) [1] <- "steps" \# rename variable

rxnmech \# print the object to be returned in the message

\# define the message that is output by the function

if (is.nu11(revrxnsist) $\{$ \{

message <- "Did not detect any reversible reactions written at two irreversible reactions."

\}else\{

message <- 1ist

paste("Detected", nrow(revrxns1st), "reversible reactions 1isted as two irreversable reactions:"),

revrxns1stmsg,

"Final reaction mechanism:",

\}

rxnmech)

\})

message \# print the message

revrxnrem <- reactive \{

\# everthing here is duplicated from the previous "revrxnmessage" function; comments above with the exception that

\# the only object that is printed is the newly created data fram itself, not the message

rxnmech <- input\$rxn

rxnmech <- data.frame(Steps = un1ist(strsplit(rxnmech, "\n")[[1]]), stringsAsFactors = FALSE)

indreacta11 <- matrix(rep("BLANK", 10*nrow(rxnmech)), nrow = nrow $(\operatorname{rxnmech}))$ 


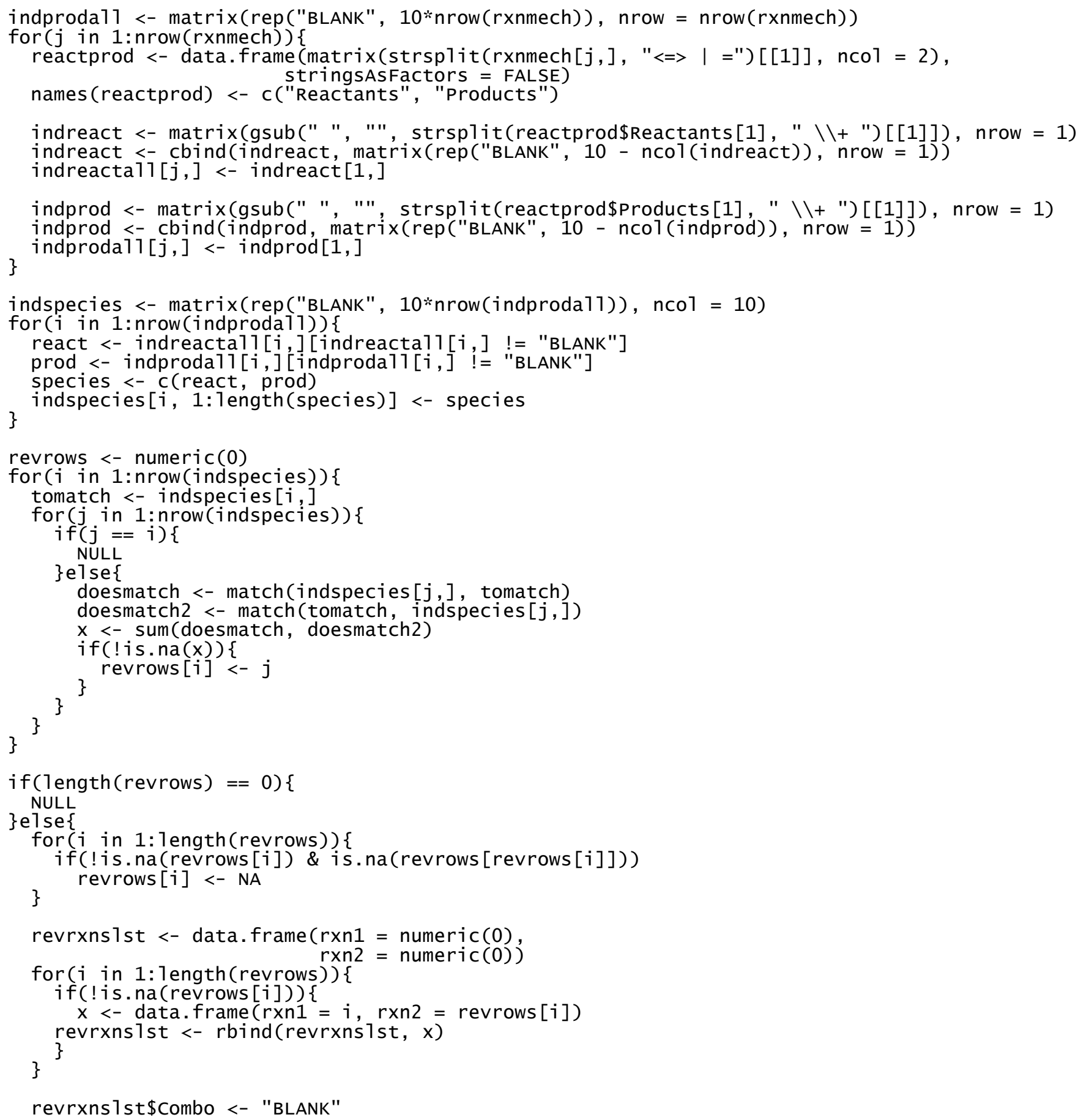


for ( $i$ in 1:nrow(revrxns1st)) \{

rxn1 <- revrxns1st\$rxn1[i]

rxn2 <- revrxns1st\$rxn2[i]

rxns <- c(rxn1, rxn2) [order $(c(r \times n 1, r \times n 2))]$

revrxnslst\$Combo[i] <- paste(rxns, collapse = " ")

revrxns1st <- revrxns1st[duplicated(revrxns1st\$Combo), ]

for ( $i$ in revrxns1st $\$ r \times n 2)\{$

if $(1$ ength $(\operatorname{grep}("<=>", \operatorname{rxnmech}[i]))=,=1)\{$

Nelses

\}

rxnmech $[i]<,-\operatorname{gsub}("=", "<=>", \operatorname{rxnmech}[i]$,

rxnmech <- as.data.frame (rxnmech [-revrxns1st\$rxn1,], \} stringsAsFactors = FALSE)

\})

rxnmech

rxnmat $<-$ reactive $(\{$

rxnmech <- revrxnrem() \# run the previous function reactively to take care of reversable reactions

al1steps <- data.frame(Substance = character $($ ) ,

coef = numeric(),

Step = numeric

stringsASFactors = FALSE) \# store substance, coefficient, and step

\# the for loop that follows will go row by row and extract the stoichiometric matrix

for $(j$ in 1:nrow(rxnmech)) \{ \# for every individual "j" row...

reactprod <- data.frame(matrix(strsplit(rxnmech $[j],, "<=>\mid=")[[1]]$, nco $1=2)$,

stringsAsFactors = FALSE) \# store "jth" row and split into reactants and products
names(reactprod) <- c("Reactants", "products") \# rename variables

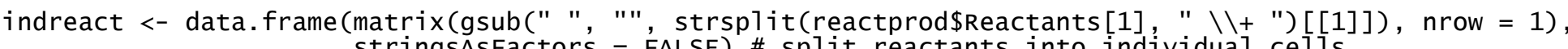
stringsAsFactor's = FALSE) \# split reactants into individual cel1s

indprod <- data.frame (matrix(gsub(" ", "", strsplit(reactprod\$Products [1], " \\+ ") [[1]]), nrow = 1), stringsAsFactors = FALSE) \# split products into individual ce11s

subname <- character() \# store the substance name

reactcoefs <- numeric() \# store the substance's (reactants) coefficient

\# the for loop that follows wi11, for every " $i$ " species in the reactants, write the substance and coefficient;

\# based on three possibilities: (1) If there is no coeffient (i.e. "H2"); (2) If there is a single digit

\# coefficient (i.e. 2H2); (3) If there is a double digit coefficient (i.e. 12H2). each one pul1s the correct

\# species coefficent

for ( $i$ in $1:$ ncol (indreact)) \{

if (is.na(as. numeric(substr (indreact $[, i], 1,1)))$ )

subname $[i]<-$ as. character (indreact $[1, i])$

reactcoefs $[i]<-1$

\}else if(! is.na(as.numeric(substr(indreact $[, i], 1,2))$ ) \& substr(indreact $[, i], 2,2) !=" E ")\{$

subname $[i]<-$ as. character (substr (indreact $[1, i], 2$, nchar (indreact $[2, i])$ ))

reactcoefs $[i]<-$ as.numeric(substr(indreact $[, i], 1,2)$ )

\}else\{

subname $[i]<-$ as.character (substr (indreact $[1, i], 2, \operatorname{nchar}(i n d r e a c t[1, i]))$ ) 
\}

reactcoefs $[i]<-$ as.numeric(substr(indreact $[, i], 1,1)$ )

reactcoefs <- data.frame(Substance = subname,

coef $=$ reactcoefs $*-1$, step $=j$,

stringsAsFactors = FALSE) \# populate the data frame

\# the if loop that follows checks if any substnaces are duplicated (i.e. $\mathrm{H}+\mathrm{H}$ should be $2 \mathrm{H}$ )

if(sum (duplicated(reactcoefs $\$$ Substance) $>0)\left\{\begin{array}{l}> \\ \text { dupsub }<- \text { reactcoefs } \$ \text { Substance [duplicated(reactcoefs } \$ \text { Substance)] \# find the duplicated substance }\end{array}\right.$

newcoef <- sum(reactcoefs\$Coef[reactcoefs\$Substance = dupsub]) \# define the new coefficient

reactcoefs <- reactcoefs [-1*match(dupsub, reactcoefs $\$$ substance), ] \# get rid of the duplicated versions

reactcoefs $\$ c 0 e f[$ reactcoefs $\$$ substance $==$ dupsub] <- newcoef \# write over existing data

\# this next section is a repeat of the previous section except for products, not reactants

subname <- character()

prodcoefs <- numeric()

for ( $i$ in $1:$ ncol (indprod)) \{

if (is.na(as.numeric(substr(indprod $[, i], 1,1))$ ) \{

subname $[i]<-$ as.character (indprod $[1, i])$ prodcoefs $[i]<-1$

\}else if(! is.na(as.numeric(substr(indprod[,i], 1, 2))) \& substr(indprod[,i], 2, 2) !="E") \{ subname $[i]<-$ as. character (substr (indprod $[1, i], 2, \operatorname{nchar}(i \operatorname{ndprod}[2, i])$ ))

\}else\{

subname [i] <- as.character (substr (indprod[1,i], 2, nchar(indprod[1,i]))) \}

prodcoefs $[i]<-$ as.numeric(substr(indprod $[, i], 1,1)$ )

prodcoefs <- data.frame(Substance = subname,

Coef $=$ prodcoefs, step $=j$

stringsAsFactors = FALSE)

if (sum (duplicated (prodcoefs $\$$ Substance)) $>0$ ) \{

dupsub <- prodcoefs $\$$ Substance [dup 1 icated (prodcoefs $\$$ Substance)]

newcoef $<-$ sum (prodcoefs $\$$ coef $[$ prodcoefs $\$$ substance $==$ dupsub])

prodcoefs <- prodcoefs [-1*match(dupsub, prodcoefs $\$$ substance), ]

\}

prodcoefs $\$$ Coef $[$ prodcoefs $\$$ substance $==$ dupsub] <- newcoef

a11steps <- rbind(a11steps, reactcoefs, prodcoefs) \# combine reactants and coefficents into data frame

substances <- unique(a11steps\$Substance) \# get the unique substances

nosubstances <<- length(substances) \# store the number of unique substances

rxnmat <- data.frame(matrix(rep(0, length (substances) * length ( $r \times n m e c h))$,

names $(r x n m a t)<-$ substances $\quad$ \# rename variables

\# the for 1oop that follows actually populates the final reaction matrix

for (i in 1:nrow(rxnmech)) \{

temp <- allsteps[a11steps $\$$ step $==i$,$] \# store the i$ th row

$x<-$ match(temp\$Substance, names(rxnmat)) \# find the substance in the data

if $(\operatorname{sum}($ duplicated $(\mathrm{x}))>0)\{$

dupsub <- data.frame(table(temp\$substance)) \# find the duplicated substance, if present

dupsub <- as. character (dupsub $\$ \operatorname{Var} 1$ [dupsub\$Freq >1]) \# find the duplicated substance, if present

temp\$Coef [temp\$Substance == dupsub] <- sum(temp\$Coef[temp\$Substance == dupsub]) \# recaculuate the coefficient

temp <- temp [! duplicated $(x)$,

\# get rid of the duplicated substances 

\} \}

rxnmat $[i, x]<-$ temp $\$$ Coef \# write to the reaction matrix

rxnmat $[$ is.na(rxnmat) $]<-0$ \# replace NAs with 0 s

\# the following section is commented out; it removes $\mathrm{H} 20$ from the mechanism

\# if(sum(substances \%in\% "H2O") > 0) \{

\# rxnmat <- rxnmat [, -match("H2O", names (rxnmat))]

$\#$ \}

nosubstances $<<-$ nosubstances -' 1

diag <- data.frame(diag(nrow(rxnmat))) \# create a diagonal matrix equal size to the reaction mechanism rxnmat <- as.matrix(cbind(rxnmat, diag)) \# add the reaction matrix \})

rxnmat
rxnmat

\# print the message

renderprint $(\{$

\})

revrxnmessage()

\# print the reaction matrix as a table

renderTable $(\{$

rxnmat()

\}, digits $=1$ )

We now perform row reduction on the above stoichiometry matrix:

$\cdots\{r$ rowred 1 , warning=FALSE, include $=$ TRUE $\}$

rowred1 <- reactive $(\{$

rxnmat <- rxnmat() \# run the function again to run in the data

rowred 1 <- data.frame $(r r e f(r x n m a t))$ \# run the row reduction and format to data frame

\# fancy way to get the number of steps

nosteps <- sum(substr(names(rowred1), 1, 1) == "X" \& ! is.na(as.numeric(substr(names(rowred1), 2, 2)))

nosubstances <- ncol (rowred1) - nosteps \# fancy way to get the number of substances

loopcheck <- rowsums (rowred1[, 1:nosubstances] != 0) \# checks if each row is a 1oop

\# names the loops

\# here, and throughout the rest of the code, there are if checks at the beginning to detects if

\# there were no loops (legal or i1lega1) in the mechanism in the first place. If there are no loops,

\# then the code simply puts out a message saying that no further code was run because there aren't

\# any loops in the mechanism.

if(1ength (c(rep(NA, nrow(rowred1) - sum (1oopcheck ==0)),

$\operatorname{nrow}($ rowred1) \{

paste0 ("L", $1: \operatorname{sum}(1$ oopcheck $==0)$ )) ) !

\}else\{

rowred1\$Loop <- $c(\operatorname{rep}(N A, \operatorname{nrow}(\operatorname{rowred} 1)-\operatorname{sum}(1$ oopcheck $==0))$ paste0 $(" L ", 1: \operatorname{sum}(1$ oopcheck $==0)))$

\}

\})

rowred1 \# print the data frame for the function's use 
From this analysis, we can identify reaction 1oops. We wil1 now only keep the data that has $100 p s$ and remove al1 reversible reactions from the next step, resulting in the second matrix prior to row reduction:

- $\{r$ rxnmat2, warning=FALSE, include $=$ TRUE $\}$

rowred1. noR <- reactive $(\{$

rowred1 <- rowred1() \# cal1 in the data after the first row reduction

\# fancy way to get the number of steps

if $(\operatorname{nrow}($ rowred1) $==1)\{$

rowred1.noR <- data.frame(Message = "No loops detected in mechanism; al1 further code wi11 not be run.") \}else\{

nosteps <- sum(substr(names(rowred1), 1, 1) == "X" \& ! is.na(as.numeric(substr(names(rowred1), 2, 2)))

nosubstances <- (ncol(rowred1) - 1) - nosteps \# fancy way to get the number of steps

loopcheck <- rowsums (rowred1[, 1:nosubstances] !=0) \# check if loops exist

rowred1.10op <- rowred1[1oopcheck ==0,-1:-nosubstances] \# reduce the data set to only contain

1oopnames <- rowred1. 1oop\$Loop \# store loop names

rxnmech <- revrxnrem() \# call in the original reaction matrix again

reversablerxns <- numeric() \# store the reversible reactions

\# the for loop that follows wi11 identify if a reaction is reversible or not

for ( $i$ in 1:nosteps)

isrev <- length $($ grep $("<=>", \operatorname{rxnmech}[i])$,$) \# find if the reaction has a "<=>"$ \}

reversablerxns[i]<- isrev' \# store it

reversablerxns <- as.logical (reversablerxns) \# convert these to logical

rowred1.noR <- rowred1. loop [, ! reversablerxns] \# remove the reversible reactions

rowred1. noR <- rowred1. noR [, ! names(rowred1.noR) \%in\% "Loop"] \# remove the "Loop" column

diag <- data.frame (diag(nrow(rowred1.noR))) \# create the new diagona1

names (diag) <- paste0("L", 1:ncol(diag)) \# rename the diagonals

rowred1.noR <- as.matrix(cbind(rowred1.noR, diag)) \# bind the data frames together

\})

rowred1.noR \# print the data for the function

rowred1. 1oop <- reactive $(\{$

\# the following sections are repeated from above until the added for loop

rowred1 <- rowred1()

if $($ nrow $($ rowred 1$)==1)\{$

loopmessage <- "No loops detected in mechanism; a11 further code wil1 not be run."

\}else\{

nosteps <- sum(substr(names(rowred1), 1, 1) == "X" \& ! is.na(as.numeric(substr(names(rowred1), 2, 2)))

nosubstances <- (ncol(rowred1) - 1) - nosteps

loopcheck <- rowsums (rowred1 [, 1: nosubstances $] !=0$ )

rowred1. 1oop <- rowred1[1oopcheck == 0, -1:-nosubstances]

rxnmech <- revrxnrem() 
reversablerxns <- numeric()

for ( $i$ in 1:nosteps)

isrev <- length $(\operatorname{grep}("<=>", \operatorname{rxnmech}[i])$,

\}

reversablerxns $[i]<-i s r e v$

reversablerxns <- as. 1ogical (reversablerxns)

rowred1. noR <- rowred1. loop [, c(!reversablerxns, TRUE)]

loopnames <- rowred1. noR \$LOop

rowred1. noR <- rowred1. noR [, !names (rowred1.noR) \%in\% "Loop"]

i11. 1oops <- numeric(0) \# store the text of the i11egal 1oops

\# the for loop that follows identifies if the loop is illegal or not

for ( $i$ in 1:nrow(rowred1.noR))

$j 11$ loop <- as vector(rowred1.noR $[i, \operatorname{rowred} 1 . \operatorname{noR}[i] !=0]$,$) \# temporarily store the i$ th row as $10 \mathrm{gica} 1$ if (1ength (i11.10op) $==0)\{$

i11. 10op $=0$ \# if all values equal 0 , it's not an illegal loop

\}else\{

$711.100 p<-$ as.vector $(i 11.100 p>0)$ \# convert TRUE/FALSE to $1 / 0$

if (sum (i11.10op) $==$ length $(i 11.100 p) \mid \operatorname{sum}(i 11.100 p)==0)\{$

i11.10op $=1$ \# if the sume of these loops is equal to the length or if the sum is 0 , that's illegal

\}else\{

\}

i11. 1oop $=0$ \# otherwise, it's not

i11.10ops[i] <- $i 11.100 p$ \# store the value

if $(\operatorname{sum}(i 11.100 p s)==0)\{$

loopmessage $<<-$ "There are no illegal loops after the first row reduction" \# message

\}else

\# the previous section only identified loops, but didn't grab the exact text; this section does this

loopnms <- loopnames[as. logical(i11.10ops)] \# store the loop names

nms <- 1ist(0) \# store the substance names

\# the for loop that follows pulls the names and coefficients of the individual reactions

for ( $i$ in 1: length(1oopnms))

1ooprowcheck <- rowred1\$Loop == 1oopnms[i] \# check if a substance is in the 1oop

looprowcheck[is.na(1ooprowcheck)] <- FALSE \# swap FALSE with NA

onerow <- rowred1[looprowcheck, ] \# keep only the one row

onerownames <- names (onerow [, onerow !=0]) \# store names of the substances

onerownames <- onerownames[! onerownames \%in\% "Loop"] \# remove "Loop"

\# round to nearest 0.5

newonerownames <- round(rowred1[1ooprowcheck, names (rowred1) \%in\% onerownames]/.5)*.5

\# paste the names together

nms $[i]]<-$ paste (paste (newonerownames, names (newonerownames), sep = ""), col1apse = " + ")

$x<-1$ ist(0) \# store the results of the total string

\# the for loop that follows pastes the final list together for the message

for ( $i$ in $1:$ length (loopnms) $)\{$

$x[[i]]<-$ paste(loopnms[i], ":", paste(unlist $($ nms $[[i]])$, collapse = " ")) 
\}$_{10 o p m e s s a g e}^{\}}$

loopmessage <<- paste ("There are", length (i11. loops [i11. Toops == 1]),

\})

\# print the message

renderPrint $(\{$

\})

rowred1. $100 p()$

\# print the row reduction without reversible reactions

renderTable $(\{$

rowred1. noR $($ )

\}, digits $=1$ )

$\cdots$

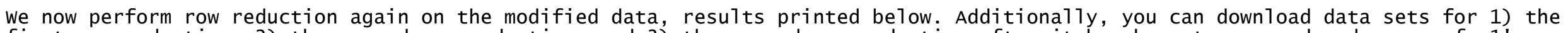

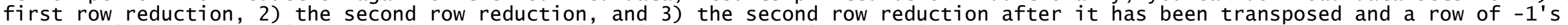
added to if for direct insertion in to Mathematica Linear Programming algorithm.

$\cdots\{r$ rowred2, warning $=F A L S E$, include $=$ TRUE $\}$

\# this function performs the 2 nd row reduction and prints the results

rowred $2<-$ reactive $(\{$

rowred1.noR <- rowred1.noR() \# cal1 in the data from previous function

if $($ nrow $($ rowred1. noR $)==1)\{$

rowred 2 <- data.frame(Message $=$ "No loops detected in mechanism; al1 further code wil1 not be run.")

\}else\{

rowred 2 <- data.frame(rref(as.matrix(rowred1.noR))) \# run the 2 nd row reduction and convert to dataframe

rowred2\$Loop <- paste0 ("L", 1:nrow(rowred2),"'") \# rename the loops

rowred2. rmrev <- rowred2

rowred2. rmrev\$Check <- 0

norows <- sum(grep1 ("x", names(rowred2)))

for ( $i$ in 1:nrow(rowred2.rmrev)) \{

check <- sum(rowred2.rmrev $[i, \operatorname{grep} 1(" X ", \operatorname{names}($ rowred2.rmrev)) $]==0)$

if (check $==$ norows) \{

$$
\text { \} }
$$

rowred $2<-$ rowred2. rmrev [rowred2. rmrev\$check $!=1$,

rowred2 <- rowred2[! names(rowred2) \%in\% "Check"]

\}

\})

rowred2 \# print to the function

\# this function performs the 2 nd row reducation and prints the message of i1legal 1oops

red2i11<- reactive $(\{$

rowred1.noR <- rowred1.noR() \# cal1 in the data from previous function

if $($ nrow $($ rowred1. noR $)==1)\{$

message <- "No loops detected in mechanism; all further code will not be run."

\}else\{ 
rowred2 <- data.frame(rref(as.matrix(rowred1.noR))) \# run the 2 nd row reduction and convert to dataframe loopnms <- paste0 ("L", 1:nrow(rowred2), "'") \# rename the loops

redtemp <- rowred2 [, substr(names(rowred2), 1, 1) != "L"] \# keep the loops on 1y

zerocheck <- $\operatorname{logical}(0)$ \# store the rows that add to 0

\# the for loop that follows tracks if the row adds to 0 or not

for ( $i$ in $1: \operatorname{nrow}($ redtemp)) \{

if $(\operatorname{sum}($ redtemp $[i]=,=0)==\operatorname{ncol}($ redtemp $))\{$

\}else\{ \}

zerocheck $[i]<-$ FALSE

redtemp <- redtemp[!zerocheck, ] \# eliminate the rows that add to 0

i11checks <- logical(0) \# store the $i 11$ egal reactions

\# the for loop that follows wil1 determine if the reaction step is illegal or not

for ( $i$ in 1:nrow(redtemp))

$111 \mathrm{check}<-\operatorname{sum}(\operatorname{redtemp}[i]<0$,

if (i11check > 0) \{

i11egal <- FALSE

\}else\{

\}

i1 1 egal <- TRUE

\}

i11checks $[i]<-i 11$ egal

redtemp $\$ 11$ legal <- $i 11$ checks \# write $i 11$ egal 1oops to the data frame

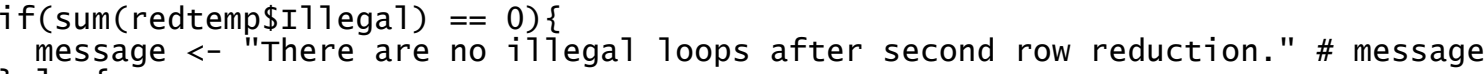
\}else\{

\# this section is similar to the prevoius section in defining a message to be printed

rowred1 <- rowred1()

rowred 2 Loop <- paste0 ("L", 1:nrow(rowred2), "'")

rowred $2 p<-\operatorname{rowred} 2[\operatorname{rownames}($ redtemp $[$ redtemp $\$ 111$ ega $1==\operatorname{TRUE}]),, \operatorname{substr}($ names $(\operatorname{rowred} 2), 1,1)==" L "]$

tnmst $<-1$ ist $(0)$

for $(i$ in $1: \operatorname{nrow}($ rowred2p) \{

$1 \mathrm{nms}<-\operatorname{names}(\operatorname{rowred} 2 \mathrm{p}[i, \operatorname{rowred} 2 \mathrm{p}[i] !=0$,$] )$

$1 \mathrm{nms}<-1 \mathrm{nms}[1 \mathrm{nms} !=$ "Loop"]

coefs <- rowred2p [i, names (rowred2p) \%in\% 1nms]

$1 \mathrm{nms} 2<-$ paste (round (coefs), $1 \mathrm{nms}$, sep="")

1nms2 <- gsub("-1", "-", 1nms2)

$1 \mathrm{nms} 2<-$ gsub("1L", "L", 1nms2)

1 nms2 <- paste (1nms2, collapse = " + ")

1nms2 <- paste(rowred2p\$Loop[i], "=", 1nms2)

nms $<-$ list $(0)$

for ( $j$ in 1: length (1nms))

looprowcheck <- rowred1\$Loop $==1 \mathrm{nms}[j]$

looprowcheck [is.na(looprowcheck)] <- FALSE

onerow <- rowred1[looprowcheck,

onerownames <- names (onerow $[$, onerow $!=0]$ ) 
onerownames <- onerownames[! onerownames \%in\% "Loop"]

newonerownames <- round(rowred1[1ooprowcheck, names(rowred1) \%in\% onerownames]/.5)*.5

$$
\text { if }(j==1)\{
$$

nms $[[j]]<-$ paste 0 (round (coefs $[j] / .5) * .5$, "(",

paste (paste (newonerownames, names (newonerownames)

$$
\text { sep = "), collapse }="+") \text {, ")") }
$$

\}e1se \{

nms $[[j]]<-$ paste 0 (round (coefs $[, j] / .5) * .5$, "(", paste (paste (newonerownames, names (newonerownames), \}$^{3}$

\} sep = "), collapse $="+")$, ")")

tmns <- paste (nms, collapse $="+$ ")

tmns <- gsub ("1\\(", "\\(", tmns)

tmns $<-$ gsub("1X", "X", tmns)
tmns $<-$ gsub("\\+ $\backslash \backslash-"$ ", "\\-", tmns) \}

tnmst $[[i]]<-\operatorname{paste}(1 \mathrm{nms} 2$, "=", tmns) \} \}

\})

message

\# this function performs and prints the second row reduction and is very similar to the first,

\# but then transforms the data as it ready to be input into Mathematica for the 1inear programming

\# sequence.

rowred2ti <- reactive $(\{$

rowred1. noR <- rowred1. noR()

if $($ nrow $($ rowred1. noR $)==1)\{$

rowred2.t1 <- data.frame(Message = "No loops detected in mechanism; a11 further code wi11 not be run.")

\}else\{

rowred $2<-$ data.frame $(\operatorname{rref}(\operatorname{rowred} 1$. noR $))$

rowred2. rmrev <- rowred2

rowred2. rmrev\$Check $<-0$

norows <- sum(grep1 ("X", names(rowred2)))

for ( $i$ in 1 : nrow(rowred2. rmrev) \{

check <- sum(rowred2.rmrev[i, grep1 ("X", names(rowred2.rmrev)) ] ==0)

if (check == norows) \{

\} \}

rowred2.rmrev\$Check $[i]<-1$

rowred $2<-$ rowred $2 . \operatorname{rmrev}[$ rowred $2 . \operatorname{rmrev} \$$ check $!=1$,

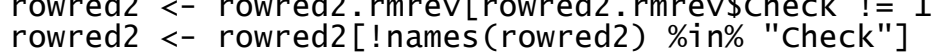

Lcols <- substr (names (rowred2), 1, 1)
rowred2. noL <- rowred2 [, LCo1s == "X"]

rowred2. noL $<-$ rowred2 [, Lcols $==" ~ " X "]$
rowred2.t $<-$ data.frame (t (rowred2. noL)

\}

rowred2.t $1<-\operatorname{rbind}(\operatorname{rowred} 2 . t, \operatorname{rep}(-1, \operatorname{ncol}(\operatorname{rowred} 2 . t)))$ 
\# print the second row reduction

renderTable $(\{$

$$
\text { rowred2() }
$$

\}, $\operatorname{digits}=1$ )

\section{\# print the message}

renderprint ( \{

\}) red2i11()

\# print the second row reduction

renderTable $(\{$

rowred2ti ()

\}, digits = 1)

\# Shiny code to program functionality and downloads

ui <- fluidpage (downloadButton ("drowred1", "Download 1st Row Reduction"),

downloadButton ("drowred2"', "Download 2nd Row Reduction")

downloadButton("drowred3", "Download Data for Linear Programming in Mathematica"))

server <- function(input, output)

output $\$$ drowred1 <- downloadHandler

filename = function() \{

\},

paste("1strowred", ".csv", sep="")

content $=$ function $(f i l e)$

) \}

write. $\operatorname{csv}(\operatorname{rowred} 1()$, file, row. names $=$ FALSE $)$

output $\$$ drowred2 <- down 1oadHandler (

filename = function() \{

\} ,

paste ("2ndrowred", ".csv", sep="")

content $=$ function $(\mathrm{fi} 1 \mathrm{e})$

) \} write.csv(rowred2(), file, row.names = FALSE)

output \$drowred3 <- downloadHandler (

filename = function() \{ \} , paste ("2ndrowredtrans", ".csv", sep="")

content = function (file) \}

write.csv(rowred2ti(), file, row.names = FALSE)

\section{\}}

shinyapp (ui, server) 


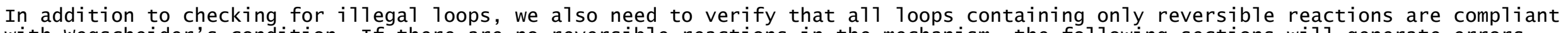

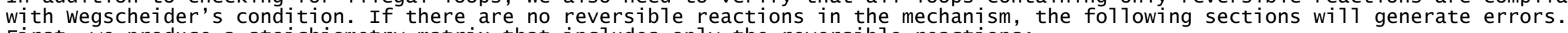
First, we produce a stoichiometry matrix that includes only the reversible reactions:

$\cdots\{r$ weg, warning=FALSE, include=TRUE $\}$

stoichmatreversable <- reactive $(\{$

stoichmatrev <- rxnmat()

rowred1 <- rowred1()

nosteps <- sum(substr(names(rowred1), 1, 1) == "X" \& ! is.na(as.numeric(substr(names(rowred1), 2, 2)))

rxnmech <- revrxnrem() \# cal1 in the original reaction matrix again

reversablerxns <- numeric() \# store the reversible reactions

\# the for loop that follows will identify if a reaction is reversible or not

for ( $i$ in 1: nosteps) \{

isrev <- length (grep $("<=>", \operatorname{rxnmech}[i])$,$) \# find if the reaction has a "<=>"$ \}

reversablerxns[i]<- isrev' \# store it

reversablerxns <- as.logical(reversablerxns)

\# convert these to logical

stoichmatrev <- stoichmatrev[reversablerxns,]

stoichmatrev

\})

renderTable ( \{

stoi chmatreversable()

\} , digits $=1$ )

…

Next, we'11, perform row reduction to identify any loops within the reversible reactions so that we can manually verify that Wegscheider's condition is met.

$\cdots\{r$ WegLoop, warning=FALSE, include=TRUE $\}$

rowred1.rev <- reactive $(\{$

rxnmat <- stoichmatreversable()

\# run the function again to run in the data

rowred1 <- data.frame(rref(rxnmat)) \# run the row reduction and format to data frame

\# fancy way to get the number of steps

nosteps <- sum(substr(names(rowred1), 1, 1) == "X" \& ! is.na(as.numeric(substr(names (rowred1), 2, 2)))

nosubstances <- ncol(rowred1) - nosteps \# fancy way to get the number of substances

loopcheck <- rowsums (rowred1 $[, 1$ : nosubstances $] !=0$ ) \# checks if each row is a loop
\# names the loops

\# here, and throughout the rest of the code, there are if checks at the beginning to detects if

\# there were no loops (1egal or i11ega1) in the mechanism in the first place. If there are no 1oops,

\# then the code simply puts out a message saying that no further code was run because there aren't

\# any loops in the mechanism.

if(length (c(rep(NA, nrow(rowred1) - sum(1oopcheck == 0)),

nrow (rowred1))

paste0 ("L", 1: $\operatorname{sum}(1$ oopcheck $==0)$ )) ) $!=$

rowred1 <- data.frame(Message = "No 1oops detected in mechanism; a11 further code wi11 not be run.")

\}else\{

rowred1\$Loop <- $c(\operatorname{rep}(N A, \operatorname{nrow}(\operatorname{rowred} 1)-\operatorname{sum}(10 o p c h e c k==0))$, 
rowred1. loop.rev <- reactive $(\{$

\# the following sections are repeated from above until the added for $100 p$ rowred $1<-$ rowred 1 . rev ()

if $($ nrow $($ rowred 1$)==1)\{$

loopmessage <- "No loops detected in mechanism; a11 further code wi11 not be run."

\}else\{

nosteps <- sum(substr(names(rowred1), 1, 1) == "X" \& ! is.na(as.numeric(substr(names (rowred1), 2, 2)))

nosubstances <- (ncol (rowred1) - 1) - nosteps

loopcheck <- rowSums (rowred1 [, 1: nosubstances] $!=0$ )

rowred1. 1oop <- rowred1[1oopcheck == 0, -1:-nosubstances]

rxnmech <- revrxnrem()

reversablerxns <- numeric()

for ( $i$ in 1:nosteps) \{

isrev $<-$ length $(\operatorname{grep}("<=>", \operatorname{rxnmech}[i])$,

\}

reversablerxns [i] <- isrev

reversablerxns <- as. 1ogical (reversablerxns)

rowred1.R <- rowred1. loop [, c(reversablerxns, TRUE)]

loopnames <- rowred1. R\$Loop

rowred1.R <- rowred1.R[, !names(rowred1.R) \%in\% "Loop"]

i11.10ops <- numeric(0) \# store the text of the illegal loops

\# the for loop that follows identifies if the loop is illegal or not

for ( $i$ in 1:nrow (rowred1. R) )

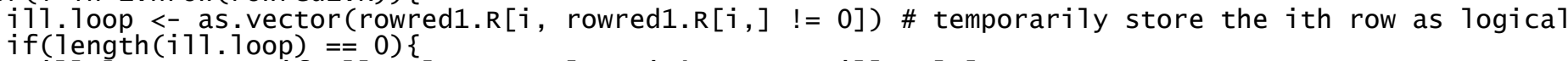

i11. 10op $=0$ \# if a11 values equal 0 , it's not an illegal loop

\}e 1 se \{

i11.10op <- as.vector (i11.10op > 0) \# convert TRUE/FALSE to $1 / 0$

if $(\operatorname{sum}(i 11.100 p)==1$ ength $(i 11.70 o p) \mid \operatorname{sum}(i 11.100 p)==0)\{$

i11.10op = 1 \# if the sume of these loops is equal to the length or if the sum is 0 , that's illegal \}e 1 se \{

i11.7oops [i] <- i11.7oop \# store the value \}

111. 1 oop $=0$ \# otherwise, it's not

\# the previous section only identified loops, but didn't grab the exact text; this section does this 1oopnms <- loopnames \# store the loop names

nms <- 1ist(0) \# store the substance names

\# the for loop that follows pulls the names and coefficients of the individual reactions

for ( $i$ in 1: length(loopnms)) \{

looprowcheck $<-$ rowred1\$Loop == 1oopnms[i] \# check if a substance is in the 1oop

looprowcheck[is.na(looprowcheck)] <- FALSE \# swap FALSE with NA

onerow <- rowred1[looprowcheck,] \# keep only the one row

onerownames <- names (onerow $[$, onerow !=0]) \# store names of the substances 
onerownames <- onerownames[! onerownames \%in\% "Loop"] \# remove "Loop" \# round to nearest 0.5

newonerownames <- round(rowred1[1ooprowcheck, names(rowred1) \%in\% onerownames]/.5)*.5

\# paste the names together

nms $[[i]]<-$ paste (paste(newonerownames, names (newonerownames), sep = ""), col1apse = " + ")

$x<-$ list(0) \# store the results of the total string

\# the for loop that follows pastes the final list together for the message

for ( $i$ in $1:$ length (1oopnms) \{

\}

$x[[i]]<-$ paste(loopnms[i], ":", paste(unlist(nms[[i]]), collapse = " "))

\# compose the final message

loopmessage $<-$ paste("There are", length(i11.10ops),

\}

\})

loopmessage

renderprint $(\{$

\})

rowred1.10op.rev()

renderTable ( \{

rowred1. rev(

\}, digits = 1)

, 